\title{
Urabstimmung FMH Services
}

\section{Einleitung}

Wie auch im vergangenen Jahr hat die Verwaltung der FMH Services beschlossen, die statutarischen Geschäfte (Abnahme des Jahresberichtes, Genehmigung der Rechnung 2002 sowie Dechargeerteilung an die Verwaltung) einer Urabstimmung zu unterziehen. Die notwendigen Unterlagen sind nachfolgend in dieser Nummer der Schweizerischen Ärztezeitung publiziert. Die genauen Modalitäten sind nachfolgend beschrieben.

\section{Wer ist stimmberechtigt?}

Stimmberechtigt sind alle FMH-Mitglieder, welche vor dem 31. Dezember 2001 in die FMH als ordentliches oder ausserordentliches Mitglied aufgenommen worden sind. Weiter alle FMHMitglieder, welche sich beim Eintritt in die FMH auch zum Beitritt in die Genossenschaft FMH Services entschlossen haben.

\section{Wie wird abgestimmt?}

Alle Unterlagen für die Urabstimmung sind nachfolgend publiziert. Alle Anträge sind auf einem separaten Stimmzettel zusammengefasst. Der ausgefüllte Stimmzettel wird im Stimmcouvert in das beiliegende Rückantwortcouvert gesteckt und an die bezeichnete Treuhandstelle geschickt. Um gültig zu stimmen, ist es wichtig, das Rückantwortcouvert mit Praxisstempel und persönlicher Unterschrift zu versehen. Nur so kann gewährleistet werden, dass ausschliesslich Genossenschaftsmitglieder ihre Stimme abgeben.

\section{Welche Frist ist zu beachten?}

Die Stimmzettel müssen bis spätestens 30. August 2003 bei der Treuhandstelle eingetroffen sein.

\section{Wer erteilt Auskünfte?}

Für Fragen steht der Geschäftsleiter der FMH Services, Markus Baumgartner, unter der Telefonnummer 0419250077 gerne zu Ihrer Verfügung.

Für die Verwaltung der FMH Services

Dr. med. H.H. Brunner

Dr. med. M. Giger Präsident Vizepräsident 


\section{Votation par correspondance FMH Services}

\section{Introduction}

A l'instar de l'année précédente, l'administration de FMH Services a décidé de soumettre les affaires statutaires (approbation du rapport annuel et des comptes 2002, ainsi que l'attribution d'une décharge à l'administration) à une votation générale. Les documents nécessaires sont publiés dans le présent numéro du Bulletin des médecins suisses. Les modalités de détail figurent ci-après.

\section{Qui a le droit de vote?}

Ont le droit de vote tous les membres FMH qui ont été admis comme membres ordinaires ou extraordinaires avant le 31 décembre 2001, ainsi que tous les membres FMH qui lors de leur entrée à la FMH ont souhaité d'acquérir aussi la qualité d'associé de la société FMH SERVICES.

\section{Comment la votation se déroule-t-elle?}

Tous les documents nécessaires pour la votation sont publiés ci-dessous. Un bulletin de votation contient l'ensemble des propositions. Le bulletin complété sera mis dans l'enveloppe de votation et celle-ci dans l'enveloppe réponse ci-jointe qui sera ensuite envoyée à l'agence fiduciaire indiquée. Pour que le vote soit valable l'enveloppe réponse doit être pourvue de cachet officiel et de signature personnelle. Ceci pour assurer que seuls les associés ne participent à la votation.

\section{Quels sont les délais?}

Les bulletins de votation doivent arriver à l'agence fiduciaire le 30 août 2002 au plus tard.

\section{Qui peut donner des renseignements?}

Le directeur de FMH Services, Markus Baumgartner, sera heureux de répondre à vos questions. Son numéro de téléphone est 0419250077.

Pour l'administration de FMH Services

Dr H. H. Brunner

Président

Dr M. Giger Vice-président 


\section{FMH Services - Jahresbericht 2002}

Markus Baumgartner, Geschäftsführer FMH Services

Mit der Verfügung eines befristeten Zulassungsstopps fiel im Berichtsjahr 2002 ein folgenschwere Entscheidung der Politiker zu Lasten der Jungärzte. Da die Massnahme lange diskutiert wurde, ist es aber dem Grossteil der Ärztinnen und Ärzte, welche in den nächsten Jahren eine Praxis eröffnen wollen, gelungen, eine Praxisbewilligung zu erhalten. Vielen konnte bei Unsicherheiten durch die FMH Services Praxisberatung und den Rechtsdienst der FMH geholfen werden.

Die bürokratischen Hürden, speziell natürlich der Zulassungsstopp, bildeten den Hauptdiskussionspunkt anlässlich der FMH-ServicesSeminare. Wir konnten auch im vergangenen Jahr wieder feststellen, dass praktisch jeder Praxiseröffner an mindestens einem unserer Seminare teilgenommen hat. Die zentrale Bedeutung unsere Informationsplattformen für die Praxiseröffnung spiegelt sich auch in der sehr guten Benotung der Seminarinhalte und deren Referenten wider.

Nebst der Begleitung bei der Praxiseröffnung steht für die Praxisberatung auch die Begleitung von Mitgliedern bei der Praxisauf- oder -übergabe im Vordergrund. Die auf realistischen Werten basierenden Inventarwertschätzungen und Goodwillberechnungen sind nach wie vor ein gefragtes Instrument, um den Verkaufswert einer
Praxis zu bestimmen. Bei den Praxiseröffnungen wichtig sind auch die Musterverträge für Miete und Anstellung von Personal. Diese Verträge können in enger Zusammenarbeit mit dem Rechtsdienst der FMH individuell den Ansprüchen der einzelnen Ärztinnen und Ärzte angepasst werden.

Die «Aussenstellen» der FMH Services erfuhren im vergangenen Jahr einen massiven Ausbau. Die Vertrauenspartner im Bereich Versicherung und Finanzanlagen stehen nun in 21 Orten in der ganzen Schweiz für die Ärzteschaft zur Verfügung. Die Produkte werden laufend dem Markt angepasst und stehen exklusiv den Vertrauenspartnern der FMH Services offen.

Ebenfalls mit 21 Partnern steht die FMH Services Treuhand praktisch flächendeckend in der ganzen Schweiz den FMH-Mitgliedern zur Verfügung. Im laufenden Jahr werden noch bestehende Lücken geschlossen werden.

Die Arbeiten an der Online-Stellenvermittlung wurden im vergangenen Jahr in Angriff genommen. Das Projekt wurde mit der Aufschaltung des Angebotes (www.fmhjob.ch) in diesem Jahr abgeschlossen.

Wie der Geschäftsabschluss zeigt, bewegen sich die FMH Services auch finanziell im vorgesehenen Rahmen. 


\section{Bilanz per 31. Dezember 2002}

\author{
AKTIVEN \\ Umlaufvermögen \\ Bankguthaben \\ Wertschriften \\ Forderungen gegenüber Tochtergesellschaften \\ Andere kurzfristige Forderungen gegenüber Tochtergesellschaften \\ Andere kurzfristige Forderungen \\ Anlagevermögen \\ Immobilien \\ Anteilscheine \\ Beteiligungen \\ Langfristige Forderungen gegenüber Tochtergesellschaften \\ Immobile Sachanlagen \\ Total Aktiven \\ 672599.17 \\ 256875.97 \\ 235453.20 \\ 74655.00 \\ 100000.00 \\ 5615.00 \\ 3419100.00 \\ 1209099.00 \\ 1000.00 \\ 776500.00 \\ 1405000.00 \\ 27501.00 \\ 4091699.17
}

\section{PASSIVEN}

Fremdkapital

Kurzfristige Verbindlichkeiten gegenüber Dritten

Verbindlichkeiten aus Leistungen gegenüber Nahestehenden

Andere kurzfristige Verbindlichkeiten gegenüber Tochtergesellschaften

Langfristige Finanzverbindlichkeiten

Langfristige Rückstellungen

Passive Rechnungsabgrenzung

Eigenkapital

Zuwachskapital

Allgemeine Reserven

Bilanzgewinn

Jahresgewinn

Total Passiven
3120868.47

3094088.46

26780.01

970830.70

12097.50

44733.20

120000.00

745000.00

29500.00

19500.00

091699.17 


\title{
Erfolgsrechnung 1. Januar - 31. Dezember 2002
}

\author{
Erfolg aus Liegenschaften \\ Mietzinseinnahmen \\ 137610.00 \\ $-29034.35$ \\ Hypothekarzinsaufwand \\ $-3415.35$ \\ Abgaben, Gebühren, Objektsteuer \\ $-2994.40$ \\ 102165.90 \\ Erfolg aus Finanzanlagen \\ Ertrag aus Finanzanlagen \\ 83879.91 \\ Erfolg aus Immobilien und Finanzanlagen \\ 186045.81 \\ Betriebsaufwand \\ Personalaufwand \\ 27676.00 \\ Sonstiger Betriebsaufwand \\ 94864.80 \\ Betriebsgewinn vor Abschreibungen und Steuern \\ 63505.01 \\ Steuern \\ 9004.90 \\ Betriebsgewinn vor Abschreibungen \\ 54500.11 \\ Bildung Delkredere \\ 3550.00 \\ Abschreibungen \\ 27497.10 \\ Betriebsgewinn 1.1.2002 - 31.12.2002 \\ 23453.01 \\ a. o. Ertrag \\ 3327.00 \\ Unternehmungsgewinn 1.1.2002 - 31.12.2002 \\ 26780.01
}

\section{Anhang}

Freiwillige Angaben in Anlehnung an Art. 663b OR

Bürgschaften zu Gunsten Dritter davon beansprucht

Verpfändung von Aktiven zur Sicherstellung eigener Verpflichtungen

Brandversicherungswerte der Sachanlagen

Mobile Anlagen, total Police

Immobilien

Gesamte Liegenschaft Elfenstrasse 18, Bern

2001: Fr. 4400000.00

2002: Fr. 4732600.00

davon Miteigentum FMH Services: $50 \%$

2200000.00

2366300.00

In der Police betreffend mobile Anlagen sind die mobilen Anlagen der FMH Inkasso Services und der FMH Consulting Services mitversichert und somit im Brandversicherungswert enthalten.

Bericht der Kontrollstelle

Die Kontrollstelle PriceWaterhouseCoopers hat die Rechnung geprüft und empfiehlt sie der Urabstimmung zur Annahme.
31. 12.2002

590000.00

409914.71

1209099.00

2366300.00

865850.00 


\section{FMH Services - Rapport annuel 2002}

Markus Baumgartner, directeur de FMH Services

En décrétant le blocage de l'accès à la pratique privée pour une durée déterminée, les politiciens ont pris une décision lourde de conséquences pour les jeunes médecins. En raison des longues discussions ayant mené à l'introduction de cette mesure, la plupart des médecins qui souhaitaient ouvrir un cabinet ces prochaines années ont tout de même pu obtenir une autorisation durant ce laps de temps. En cas d'incertitudes, nombreux sont ceux qui ont eu recours aux conseils de FMH Services pour toutes les questions concernant l'ouverture d'un cabinet médical.

Les obstacles administratifs, en particulier le blocage précité, ont constitué les principauxobjets de discussions au cours des séminaires organisés par FMH Services. Par ailleurs, nous avons une nouvelle fois constaté au cours de l'exercice écoulé que presque chaque nouveau détenteur de cabinet médical avait participé à au moins un de nos séminaires. En outre, les excellents échos que nous avons reçus en ce qui concerne le contenu des cours et les intervenants ont aussi permis de démontrer l'importance capitale de nos plates-formes d'information consacrées à l'ouverture d'un cabinet médical.

Outre l'assistance lors de l'ouverture d'un cabinet médical, nous aidons également les membres FMH en cas de remise ou reprise d'un cabinet. Le calcul de la valeur d'inventaire et du goodwill basé sur des données réelles sont très demandés pour déterminer la valeur de vente d'un cabinet médical. En cas d'ouverture de cabinet, les modèles de contrat concernant les baux à loyer et l'engagement du personnel médical sont également très utiles. Ces contrats peuvent, en étroite collaboration avec le service juridique de la FMH, être adaptés aux besoins individuels de chaque médecin.

Les «services externes» de FMH Services ont été grandement étendus. Nos partenaires de confiance dans le domaine des assurances et des investissements financiers sont désormais à la disposition du corps médical dans 21 localités de Suisse. Nos produits sont constamment adapté au marché et sont exclusivement réservés à ces partenaires de confiance.

La fiduciaire FMH Services, dotée elle aussi de 21 partenaires, sert également les membres FMH dans presque toute la Suisse. L'année en cours permettra de combler les lacunes restantes.

Les travaux pour la mise sur pied d'un service de placement en ligne ont été lancés l'année dernière. Le projet est désormais achevé et peut être consulté sur l'internet (www.fmhjob.ch).

La clôture de l'exercice a en outre montré que FMH Services se trouvait dans le cadre prévu sur le plan financier. 


\section{Bilan au 31 décembre 2002}

\author{
ACTIFS \\ Capital de roulement \\ Banques \\ Titres et valeurs \\ Créances sur prestations envers des tiers \\ Autres créances à court termes envers des organisations affiliées \\ Autres créances à court termes \\ Capital investi \\ Immeubles \\ Titres \\ Participations \\ Créances à long termes envers des organisations affiliées \\ Immobiliers \\ Total ACTIFS \\ 4091699.17
}

\section{PASSIFS}

Capitaux confiés

970830.70

12097.50

44733.20

120000.00

745000.00

29500.00

19500.00

Provisions à long terme

Passifs de régularisation

3120868.47

Capital effectif

3094088.46

Capital

Réserve générale

Gain du bilan

Gain d'exploitation

26780.01

Total PASSIFS 


\section{Compte d'exploitation du $1^{\text {er }}$ janvier au 31 décembre 2002}

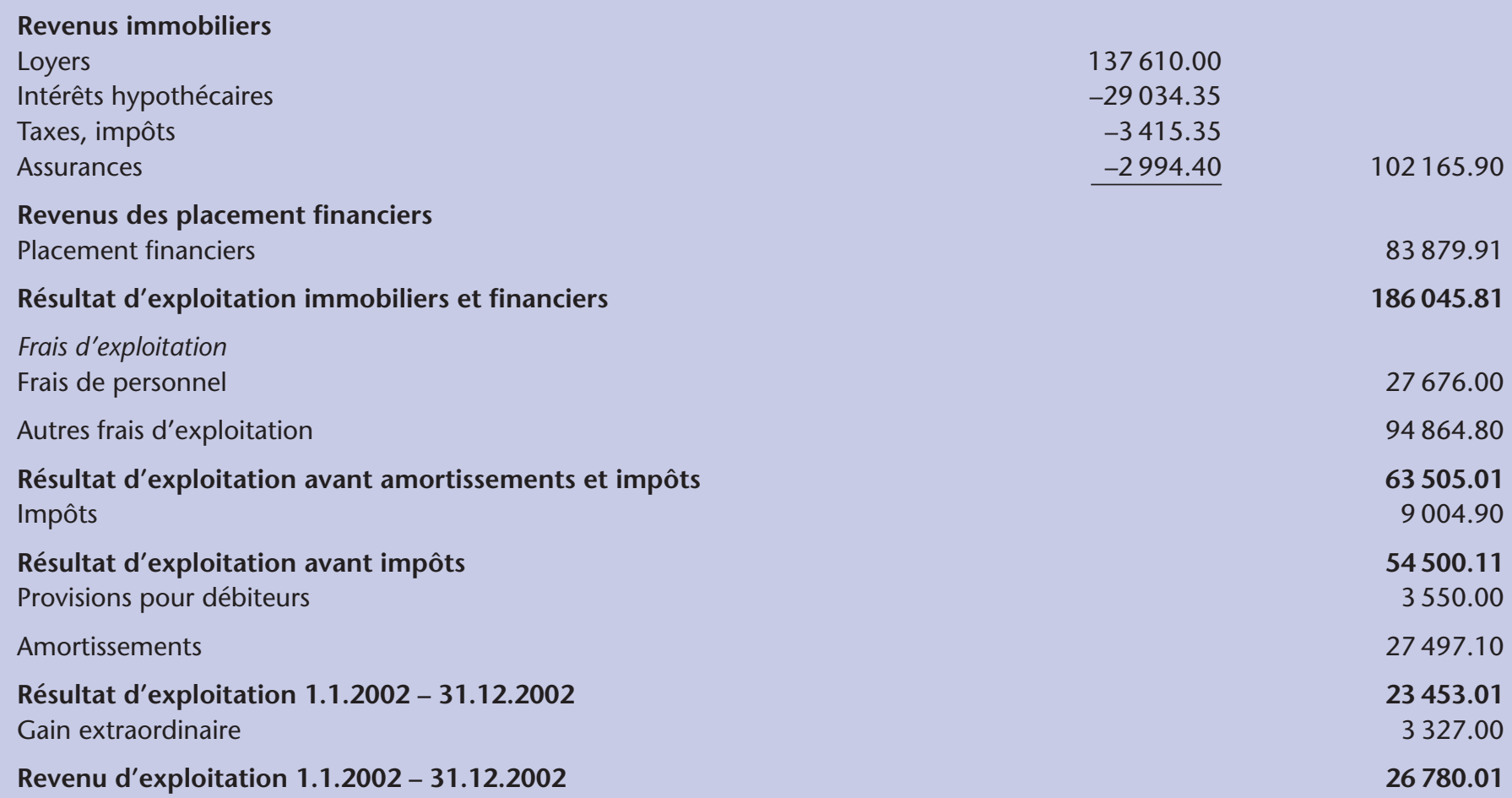

\section{Annexe}

Informations facultatives selon l'art. 663b CO

31. 12.2001

590000.00

370396.30

dont montant sollicité

Garantie d'actifs pour engagements propres

Valeurs d'assurance-incendie des immobilisations corporelle

Biens mobiliers

Biens immobiliers

Immeuble de la Elfenstrasse 18, Berne

2001: Fr. 4400000.00

2002: Fr. 4732600.00

Dont copropriété FMH Services: $50 \%$
1190000.00

3065850.00

865850.00
31. 12.2002

590000.00 409914.71

1209099.00

2366300.00 865850.00

La Police d'assurance concernant les biens mobiliers couvre également ceux de FMH Inkasso Services SA Services SA et FMH Consulting Services SA; ceux-ci sont donc compris dans la valeur d'assurance-incendie.

\section{Rapport de I'organe de contrôle}

L'organe de contrôle le PriceWaterhouseCoopers a vérifié la comptabilité et les comptes annuels et recommande approuver les comptes annuels. 